\title{
Coherent optical control of the ultrafast dephasing and mobility in a polar semiconductor
}

\author{
J. D. Lee, ${ }^{1, a)}$ H. Gomi, ${ }^{1}$ and Muneaki Hase ${ }^{2,3}$ \\ ${ }^{1}$ School of Materials Science, Japan Advanced Institute of Science and Technology, Ishikawa 923-1292, \\ Japan \\ ${ }^{2}$ Institute of Applied Physics, University of Tsukuba, 1-1-1 Tennodai, Tsukuba 305-8573, Japan \\ ${ }^{3}$ PRESTO, Japan Science and Technology Agency, 4-1-8 Honcho, Kawaguchi, Saitama 332-0012, Japan
}

(Received 16 August 2009; accepted 8 September 2009; published online 16 October 2009)

Using the nonperturbative many-body time-dependent approach, we investigate the nonequilibrium dynamics of the coherent longitudinal optical phonon-plasmon coupled (LOPC) modes in a polar semiconductor and explore their coherent optical control and eventually the carrier mobility of the semiconductor. The basic idea for a control of the carrier mobility is to manipulate the ultrafast dephasing of the coherent carrier-relevant LOPC mode. We theoretically propose two possible options to realize the idea and reach the final goal. One is to optimize a semiconductor by finely balancing two kinds of carrier densities by chemical doping and optical doping (or photodoping), where the relaxation of the coherent carrier-relevant LOPC mode would respond in a (weak) singular way. It is found that, in this way, the carrier mobility could be enhanced by a few tens of percent. The other is to optimize the optical pumping laser. In this option, the pulse train creating pure virtual carriers through the below-band-gap excitation would be incorporated for an optical pumping, which can make possible the dephasing-free dynamics of the coherent carrier-relevant LOPC mode. The carrier mobility can then be efficiently controlled and dramatically enhanced by synchronizing the pulse train with its coherent oscillation. This might imply one of ultimate ways to control the carrier mobility of the semiconductor. () 2009 American Institute of Physics. [doi:10.1063/1.3243342]

\section{INTRODUCTION}

In a polar semiconductor, the longitudinal optical (LO) phonon strongly couples to the carrier plasmon by the longranged Coulomb interaction. The charge carriers screen the phonons so that the new coupled eigenmodes, called the LO phonon-plasmon coupled (LOPC) mode, result: one is the lattice-relevant (i.e., phononlike) $L$ - mode and the other is the carrier-relevant (i.e., plasmonlike) $L+$ mode when the carrier density gives the plasmon frequency higher than the LO phonon frequency. ${ }^{1,2}$ The femtosecond ultrashort laser pulse makes it possible to probe the coherent oscillation of such modes in terahertz frequency range. ${ }^{3}$ The ultrafast dynamics of LOPC modes by the photoexcited (hot) carriers elucidates the carrier-carrier and the carrier-lattice interaction. ${ }^{4}$ Due to the reason, there have been extensive studies about the relaxation dynamics of the LOPC modes using the femtosecond time-resolved spectroscopy for $\mathrm{GaAs},{ }^{5-7} \mathrm{InP}^{8} \mathrm{GaP}^{9}$ and InN. ${ }^{10,11}$

The carrier mobility is one of the central quantities in operation of modern semiconductor devices such as high electron mobility transistor or field effect transistor or in generation and manipulation of terahertz radiation from semiconductor surfaces by the photo-Dember effect. ${ }^{12,13}$ The magnitude of the photo-Dember effect is determined by the carrier mobility, and therefore the higher the mobility, the higher the intensity of the terahertz radiation. The carrier mobility $\mu$ is given by $\mu=e \bar{\tau} / m^{*}$, where $e$ is the electron

${ }^{a)}$ Electronic mail: jdlee@jaist.ac.jp. charge, $m^{*}$ is the carrier effective mass, and $\bar{\tau}$ is the relaxation time. $\bar{\tau}$ could be obtained by terahertz time-domain spectroscopy, ${ }^{14}$ but the accessible carrier density was limited to one lower than $\mathcal{O}\left(10^{16}\right) \mathrm{cm}^{-3}$ because of the non-Drude behavior at higher carrier densities. Meanwhile, the LOPC modes are noted to have a fundamental importance in understanding transport properties of semiconductors. Raman investigation of $\mathrm{SiC}$ polytypes has shown that the linewidth of the carrier-relevant LOPC mode can be directly matched to the carrier mobility obtained from Hall measurements in the high carrier density region. ${ }^{15}$ Hase $^{16}$ recently measured the relaxation time $\bar{\tau}_{L+}$ of the coherent carrier-relevant mode (i.e., $L+$ mode) from its real-time relaxation using timeresolved electro-optic detection and time-frequency analysis in GaAs, showing that $\mu \approx e \bar{\tau}_{L+} / m^{*}$ could give a good estimation of carrier mobility.

In this paper, we propose a new pathway for controlling the coherent motion of the LOPC mode and eventually the carrier mobility $\mu$. The basic idea is to control the carrier mobility $\mu$ by manipulating the relaxation time $\bar{\tau}_{L+}$ of the carrier-relevant $L+$ mode through the formula $\mu \approx e \bar{\tau}_{L+} / m^{*}$. We find that two theoretical options are available to realize the idea and reach the final goal. One option is to optimize a semiconductor. The controlling parameters in this option are the carrier density by chemical doping $n_{\text {chem }}$ and by optical doping (or photodoping) $n_{\mathrm{opt}} \cdot n_{\text {chem }}$ and $n_{\mathrm{opt}}$ are controllable independently of each other. The total carrier density $n$ is given by a sum of two densities, that is, $n=n_{\text {chem }}+n_{\text {opt }}$. In a given polar semiconductor, the plasmon-phonon coupling constant $g$ would be determined by $n_{\text {chem }}$, while the overall 
dynamics of LOPC modes would be governed by $n$. The ultrafast relaxation of $L+$ mode is determined by the mode interplay between phonon and plasmon, which would be manipulated by controlling $n_{\text {chem }}$ and $n$ (in terms of $n_{\text {opt }}$ ). In particular, when $g>\gamma_{\mathrm{PL}}$ (an inverse of $\gamma_{\mathrm{PL}}$ defines the relaxation time of plasmon, i.e., $\bar{\tau}_{\mathrm{PL}}=1 / \gamma_{\mathrm{PL}}$ ), the critical density $n_{c}$ is found to exist and $\bar{\tau}_{L+}$ be weakly singular and get a broad maximum near $n \sim n_{c}$. $n_{c}$ is defined to be the critical carrier density leading to a divergence of $\bar{\tau}_{L+}$ within the second order perturbation theory. This means that a nontrivial manipulation of the carrier mobility over its wide bounds in such a density region is possible. The other option is to optimize the optical pumping laser. Only virtual electron-hole pairs by the below-band-gap excitation, for which the ordermade optimized pulse train should be prepared, are exploited to drive the ultrafast dynamics of LOPC modes. ${ }^{17}$ In this option, therefore, $n_{\text {opt }}$ is not incorporated. Below-band-gap excitation of semiconductors suggests a key technique to realize the dephasing-free dynamics such as efficient coherent control of spins ${ }^{18}$ and extremely stable solitons. ${ }^{19}$ ac Stark effect shows that virtual carriers by the below-band-gap excitation give rise to exactly same physical processes as real ones except that they cause no relaxation and have no lifetime. $^{20-22}$ The virtual carriers have no lifetime so that they would not cause undesirable scattering events with the coherent modes or other (virtual) carriers by the succeeding pulses. This makes the virtual excitation preserve the coherence by suppressing the dephasing sources. In particular, it is found that the carrier mobility can be dramatically enhanced by synchronizing the pulse train (i.e., interval between pulses) with the coherent oscillation of the carrier-relevant mode.

\section{MODEL AND FORMALISM}

We consider the creation of continuum carriers (electronhole pairs) by the laser irradiation, which excite phonon and plasmon in a polar semiconductor. Phonon and plasmon interact with each other through an explicit phonon-plasmon coupling $g$. The resulting Hamiltonian $\mathcal{H}\left(=\mathcal{H}_{0}+\mathcal{V}\right)$ reads

$$
\begin{aligned}
\mathcal{H}_{0}= & \sum_{\mathbf{k}} \epsilon_{\mathbf{k}}^{v} d_{\mathbf{k}}^{\dagger} d_{\mathbf{k}}+\sum_{\mathbf{k}} \epsilon_{\mathbf{k}}^{c} c_{\mathbf{k}}^{\dagger} c_{\mathbf{k}}+\sum_{\mathbf{q}} \omega_{\mathrm{LO}} a_{\mathbf{q}}^{\dagger} a_{\mathbf{q}} \\
& +\sum_{\mathbf{k}} \sum_{\mathbf{q}} M_{\mathbf{q}} c_{\mathbf{k}}^{\dagger} c_{\mathbf{k}+\mathbf{q}}\left(a_{-\mathbf{q}}+a_{\mathbf{q}}^{\dagger}\right)+\sum_{\mathbf{q}} \omega_{\mathrm{PL}} b_{\mathbf{q}}^{\dagger} b_{\mathbf{q}} \\
& +\sum_{\mathbf{k}} \sum_{\mathbf{q}} V_{\mathbf{q}} c_{\mathbf{k}}^{\dagger} c_{\mathbf{k}+\mathbf{q}}\left(b_{-\mathbf{q}}+b_{\mathbf{q}}^{\dagger}\right)+g \sum_{\mathbf{q}}\left(a_{\mathbf{q}}+a_{-\mathbf{q}}^{\dagger}\right)\left(b_{-\mathbf{q}}\right. \\
& \left.+b_{\mathbf{q}}^{\dagger}\right), \\
\mathcal{V}= & \frac{1}{2} \sum_{\mathbf{k}} \Omega_{0} e^{i \omega \tau} \bar{\Theta}(\tau) c_{\mathbf{k}}^{\dagger} d_{-\mathbf{k}}^{\dagger}+\text { H.c. }
\end{aligned}
$$

$d_{\mathbf{k}}^{\dagger}\left(d_{\mathbf{k}}\right)$ is the hole operator for the valence band $\epsilon_{\mathbf{k}}^{v}$ and $c_{\mathbf{k}}^{\dagger}\left(c_{\mathbf{k}}\right)$ is the electron operator for the conduction band $\epsilon_{\mathbf{k}}^{c}$, while $a_{\mathbf{q}}^{\dagger}\left(a_{\mathbf{q}}\right)$ is the operator for the LO phonon $\omega_{\mathrm{LO}}$ and $b_{\mathbf{q}}^{\dagger}\left(b_{\mathbf{q}}\right)$ for the plasmon $\omega_{\mathrm{PL}} \cdot \omega_{\mathrm{PL}}$ is determined by $\sqrt{4 \pi n e^{2} / \varepsilon_{\infty} m^{*}}$ with the carrier density $n$. The excited carrier interacts with the LO phonon via Fröhlich coupling $M_{\mathbf{q}}=\sqrt{2 \pi \omega_{\mathrm{LO}}}\left(1 / \varepsilon_{\infty}\right.$ $\left.-1 / \varepsilon_{0}\right)^{1 / 2} /|\mathbf{q}|$ and also interacts with the bosonic plasmon via Lundqvist form $V_{\mathbf{q}}=\sqrt{2 \pi \omega_{\mathrm{PL}}}\left(1 / \varepsilon_{\infty}\right) /|\mathbf{q}|,{ }^{23}$ where $\varepsilon_{0}$ and $\varepsilon_{\infty}$ are low- and high-frequency dielectric constants. $\mathcal{V}$ is from the light-matter interaction by the pumping pulse. The strength of the light-matter coupling is quantified by $\Omega_{0}\left(=d_{c v} A\right)$, where $d_{c v}$ is the dipole matrix and $A$ is the field amplitude of the laser. $\bar{\Theta}(\tau)$ simulates the pulse shape and $\omega$ is the energy of the optical pumping pulse. They are laser parameters which are taken differently depending on the option for the coherence control.

For a theoretical description of the nonequilibrium ultrafast dynamics of a semiconductor, it is a powerful idea to solve the time-dependent Schrödinger equation in the manybody Hilbert space spanning the whole system. ${ }^{24}$ Dynamics is described by a single quantum state $|\Psi(\tau)\rangle$ with $A \rightarrow 0$ (i.e., $\Omega_{0} \rightarrow 0$ ),

$$
\begin{aligned}
|\Psi(\tau)\rangle= & C(\tau)|0\rangle_{c}|0\rangle_{v}|0\rangle_{\mathrm{LO}}|0\rangle_{\mathrm{PL}}+\sum_{\mathbf{q}} C_{\mathbf{q}}^{\mathrm{LO}}(\tau) \\
& \times|0\rangle_{c}|0\rangle_{v}|\mathbf{q}\rangle_{\mathrm{LO}}|0\rangle_{\mathrm{PL}}+\sum_{\mathbf{q}} C_{\mathbf{q}}^{\mathrm{PL}}(\tau) \\
& \times|0\rangle_{c}|0\rangle_{v}|0\rangle_{\mathrm{LO}}|\mathbf{q}\rangle_{\mathrm{PL}}+\sum_{\mathbf{k} \mathbf{k}^{\prime}} C_{\mathbf{k k}^{\prime}}(\tau) \\
& \times|\mathbf{k}\rangle_{c}\left|\mathbf{k}^{\prime}\right\rangle_{v}|0\rangle_{\mathrm{LO}}|0\rangle_{\mathrm{PL}}+\sum_{\mathbf{k} \mathbf{k}^{\prime}} \sum_{\mathbf{q}} C_{\mathbf{k k}^{\prime} \mathbf{q}}^{\mathrm{LO}}(\tau) \\
& \times|\mathbf{k}\rangle_{c}\left|\mathbf{k}^{\prime}\right\rangle_{v}|\mathbf{q}\rangle_{\mathrm{LO}}|0\rangle_{\mathrm{PL}}+\sum_{\mathbf{k} \mathbf{k}^{\prime}} \sum_{\mathbf{q}} C_{\mathbf{k k}^{\prime} \mathbf{q}}^{\mathrm{PL}}(\tau) \\
& \times|\mathbf{k}\rangle_{c}\left|\mathbf{k}^{\prime}\right\rangle_{v}|0\rangle_{\mathrm{LO}}|\mathbf{q}\rangle_{\mathrm{PL}} .
\end{aligned}
$$

For $|\Psi(\tau)\rangle$, we restricted the many-body Hilbert space to contain just a single coherent phonon or plasmon. In $|\Psi(\tau)\rangle$, we denote $|\mathbf{k}\rangle_{c}=c_{\mathbf{k}}^{\dagger}|0\rangle_{c}, \quad|\mathbf{k}\rangle_{v}=d_{\mathbf{k}}^{\dagger}|0\rangle_{v}, \quad|\mathbf{q}\rangle_{\mathrm{LO}}=a_{\mathbf{q}}^{\dagger}|0\rangle_{\mathrm{LO}}$, and $|\mathbf{q}\rangle_{\mathrm{PL}}=b_{\mathbf{q}}^{\dagger}|0\rangle_{\mathrm{PL}}$ for single particle states. The initial state $|\Psi(0)\rangle$ should be the ground state, i.e., $|\Psi(0)\rangle$ $=|0\rangle_{c}|0\rangle_{v}|0\rangle_{\mathrm{LO}}|0\rangle_{\mathrm{PL}}$. The time-dependent Schrödinger equation $i \partial / \partial \tau|\Psi(\tau)\rangle=\mathcal{H}|\Psi(\tau)\rangle$ results in infinitely coupled differential equations among coefficients. The transient reflectivity change $\Delta R / R$ is the actual experimental attainment from an electro-optic detection combined with a standard pump-probe method. ${ }^{3,16}$ It would phenomenologically give $\Delta R / R \propto r_{L} P_{L}+r_{E} P_{E},{ }^{25}$ where $P_{L}$ and $P_{E}$ are lattice and electronic polarizations, respectively, and $r_{L}$ and $r_{E}$ are their weight coefficients. From the explicit solution, we can calculate the transient polarizations from $P_{L}(\tau)=\langle\Psi(\tau)| \Sigma_{\mathbf{q}}\left[a_{\mathbf{q}}\right.$ $\left.+a_{\mathbf{q}}^{\dagger}\right]|\Psi(\tau)\rangle$ and $P_{E}(\tau)=\left\langle\Psi(\tau)\left|\Sigma_{\mathbf{q}}\left[b_{\mathbf{q}}+b_{\mathbf{q}}^{\dagger}\right]\right| \Psi(\tau)\right\rangle$. When we define $\mathcal{P}_{L}(\tau)$ and $\mathcal{P}_{E}(\tau)$ as

$$
\begin{aligned}
& \mathcal{P}_{L}(\tau)=\sum_{\mathbf{q}} C^{*}(\tau) C_{\mathbf{q}}^{\mathrm{LO}}(\tau)+\sum_{\mathbf{k} \mathbf{k}^{\prime} \mathbf{q}} C_{\mathbf{k k}^{\prime}}^{*}(\tau) C_{\mathbf{k k}^{\prime} \mathbf{q}}^{\mathrm{LO}}(\tau), \\
& \mathcal{P}_{E}(\tau)=\sum_{\mathbf{q}} C^{*}(\tau) C_{\mathbf{q}}^{\mathrm{PL}}(\tau)+\sum_{\mathbf{k} \mathbf{k}^{\prime} \mathbf{q}} C_{\mathbf{k k}^{\prime}}^{*}(\tau) C_{\mathbf{k k}^{\prime} \mathbf{q}}^{\mathrm{PL}}(\tau),
\end{aligned}
$$

we have $P_{L}(\tau)=2 \operatorname{Re}\left[\mathcal{P}_{L}(\tau)\right]$ and $P_{E}(\tau)=2 \operatorname{Re}\left[\mathcal{P}_{E}(\tau)\right]$.

In the Hamiltonian $\mathcal{H}$, we include only the dynamics of electrons since we consider $n$-type GaAs. Thus it is expected that the hole would not affect much the relevant dynamics. Further we impose an approximation of an infinitely massive 

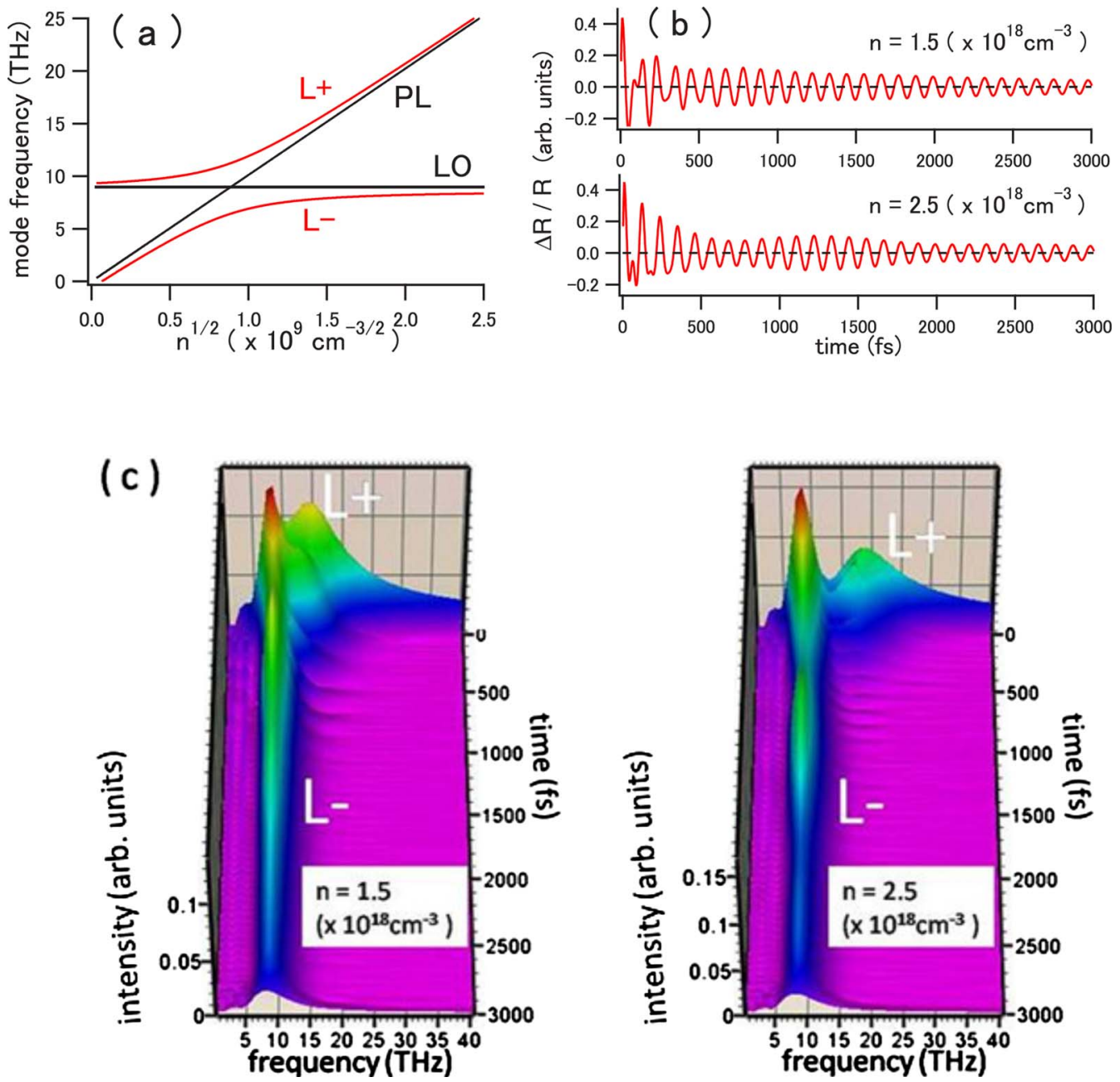

FIG. 1. (Color online) (a) LOPC modes ( $L-$ and $L+$ in red lines) for $g=10 \mathrm{meV}$. Bare phonon (LO) and plasmon (PL) are in black lines. (b) Transient electro-optic response for the reflectivity change, i.e., $\Delta R / R \propto P_{L}(\tau)+P_{E}(\tau)$, where $r_{L} / r_{E}=1$ is simply taken for the carrier densities $n=1.5 \times 10^{18}$ and 2.5 $\times 10^{18} \mathrm{~cm}^{-3}$. (c) Continuous wavelet transformation of $\Delta R / R$ in the frequency-time space for $n=1.5 \times 10^{18}$ and $2.5 \times 10^{18} \mathrm{~cm}^{-3}$.

hole by simply taking a value of the energy gap $\epsilon_{G}$ for $\epsilon_{\mathrm{k}}^{v}$, i.e., $\epsilon_{\mathrm{k}}^{v}=1.4 \mathrm{eV}$. In addition, we note $\omega_{\mathrm{LO}}=8.8 \mathrm{THz}, \epsilon_{\mathrm{k}}^{c}$ $=\mathbf{k}^{2} / 2 m^{*} \quad\left(m^{*}=0.07\right), \quad \varepsilon_{\infty}=11.3, \quad \varepsilon_{0}=13.3, \quad$ and $\quad \omega_{\mathrm{PL}}$ $=\sqrt{4 \pi n e^{2} / \varepsilon_{\infty} m^{*}}$ with the carrier density $n$. In order to incorporate the free relaxation of the phonon and plasmon without any external control, ${ }^{26}$ we simply replace $\omega_{\mathrm{LO}} \rightarrow \omega_{\mathrm{LO}}+i \gamma_{\mathrm{LO}}$ and $\omega_{\mathrm{PL}} \rightarrow \omega_{\mathrm{PL}}+i \gamma_{\mathrm{PL}}$. We define the relaxation times $\bar{\tau}_{\mathrm{LO}}$ $\equiv 1 / \gamma_{\mathrm{LO}}$ and $\bar{\tau}_{\mathrm{PL}} \equiv 1 / \gamma_{\mathrm{PL}} \cdot \bar{\tau}_{\mathrm{LO}} \gg \bar{\tau}_{\mathrm{PL}}$ is typically expected and $\bar{\tau}_{\mathrm{LO}}=2.5 \mathrm{ps}$ and $\bar{\tau}_{\mathrm{PL}}=150 \mathrm{fs}$ are fixed in our investigation unless mentioned otherwise. ${ }^{27}$ In the density range of our interest, $n>n_{0}\left(n_{0}\right.$ is defined to give $\omega_{\mathrm{PL}}=\omega_{\mathrm{LO}} ; n_{0}=0.75$ $\left.\times 10^{18} \mathrm{~cm}^{-3}\right)$, the density dependence of $\gamma_{\mathrm{PL}}$ is tiny. ${ }^{28}$

\section{COHERENT OPTICAL CONTROL OF CARRIER DEPHASING AND MOBILITY}

\section{A. First option: Optimization of semiconductor}

Under this option, we initiate the ultrafast dynamics of phonon and plasmon with a single optical pulse. $\bar{\Theta}(\tau)$ in Eq. (1) is then given by $\bar{\Theta}(\tau)=\Theta(\tau)-\Theta(\tau-\sigma)[\Theta(\tau)$ : Heaviside step function], where $\sigma$ is the length of a single pulse and fixed as $\sigma=10$ fs. $\omega=4.4 \mathrm{eV}$ is taken. As shown in Fig. 1(a), the coupling between phonon and plasmon results in new eigenmodes, i.e., LOPC modes: the lattice-relevant (i.e., phononlike) $L$ - mode and the carrier-relevant (i.e., plasmonlike) $L+$ mode under the density range of $n>n_{0}$. Energies of LOPC modes are given by $\omega_{L \pm}=\frac{1}{2}\left(\omega_{\mathrm{PL}}\right.$ $\left.+\omega_{\mathrm{LO}}\right) \pm \frac{1}{2} \sqrt{\left(\omega_{\mathrm{PL}}-\omega_{\mathrm{LO}}\right)^{2}+4 g^{2}}$. In Figs. 1(b) and 1(c), we provide the reflectivity change $\Delta R / R\left(=P_{L}+P_{E}\right.$ by taking $\left.r_{L} / r_{E}=1\right)$ and its continuous wavelet transformation in the frequency-time space for $n=1.5 \times 10^{18}$ and $2.5 \times 10^{18} \mathrm{~cm}^{-3}$, which could be directly compared to the experiment. ${ }^{16}$ In the calculation for Figs. 1(b) and 1(c), the additional isolated LO phonon has been trivially added to describe the uncoupled one in the surface depletion layer [not explicitly appears in Eqs. (1) and (2)] in contrast to LOPC modes in the bulk. ${ }^{29}$ The beating can then be observed in the phonon branch centered around 8 and $9 \mathrm{THz}$, which is induced by the coexistence of bare LO phonon and $L$ - mode. From the beating period, we could estimate the phonon-plasmon coupling constant $g$. According to Fig. 1, it is confirmed that $g$ $=10 \mathrm{meV}$ could give a beating period of about $1.25 \mathrm{ps}$ for $n=2.5 \times 10^{18} \mathrm{~cm}^{-3}$.

It is our main concern to investigate the relaxation time of LOPC modes. In Fig. 2, transient polarizations of LOPC modes are illustrated in comparison with bare phonon and 

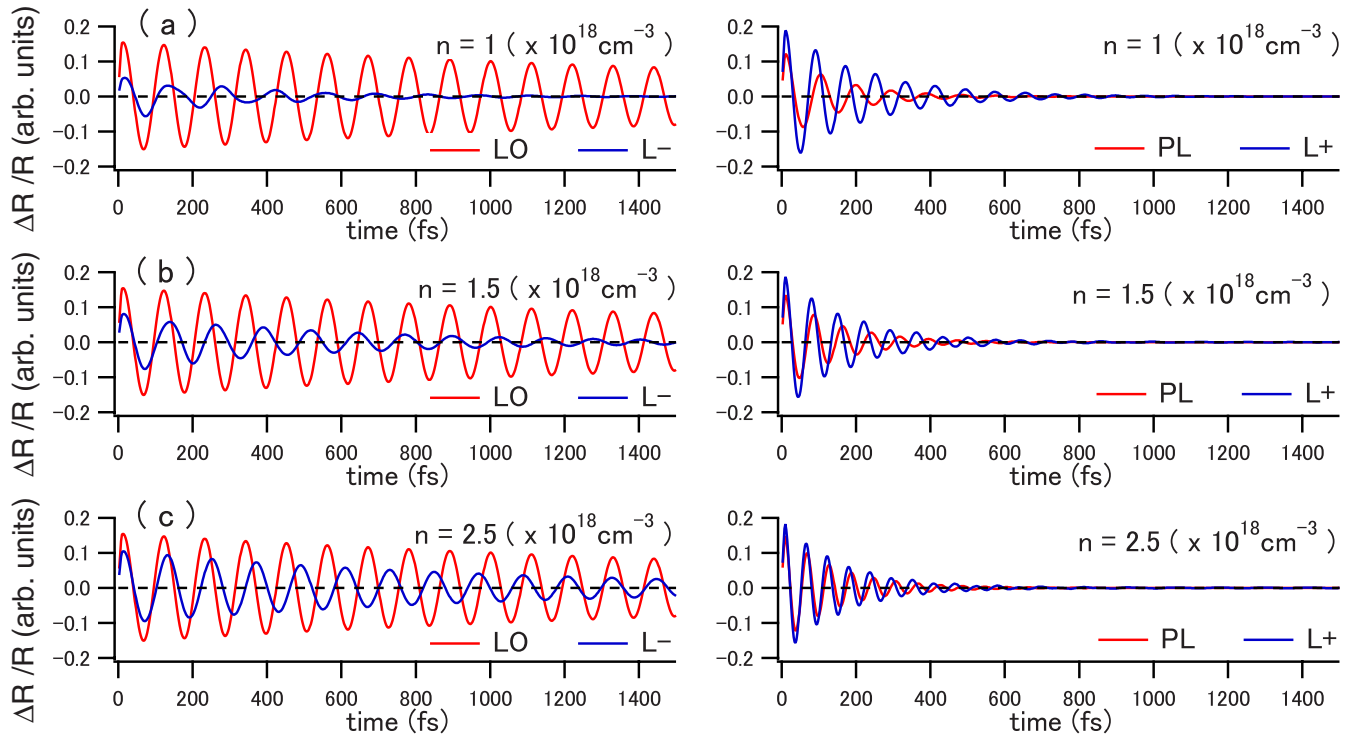

FIG. 2. (Color online) Transient polarizations of bare phonon (LO) and bare plasmon (PL) and lattice-relevant $(L-)$ and carrier-relevant $(L+)$ LOPC modes with $g=10 \mathrm{meV}$ with respect to the carrier densities $n=1 \times 10^{18}, 1.5 \times 10^{18}$, and $2.5 \times 10^{18} \mathrm{~cm}^{-3}$.

bare plasmon, $P_{L+}(\tau)=\cos \theta P_{L}(\tau)+\sin \theta P_{E}(\tau)$ and $P_{L-}(\tau)$ $=-\sin \theta P_{L}(\tau)+\cos \theta P_{E}(\tau)$, where $\tan 2 \theta=-2 g /\left(\omega_{\mathrm{PL}}-\omega_{\mathrm{LO}}\right)$. The relaxation times of $L+$ and $L-$ modes are determined directly from the temporal propagation of $P_{L_{+}}(\tau)$ and $P_{L_{-}}(\tau)$, which are found to be strongly affected by the mode coupling and screening interplay between phonon and plasmon. The relaxation time of $L+$ mode $\bar{\tau}_{L+}$ gets larger than the bare plasmon mode, but the relaxation time of $L$ - mode $\bar{\tau}_{L-}$ gets smaller than the bare phonon mode. Further, as the carrier density decreases from $n=2.5 \times 10^{18} \mathrm{~cm}^{-3}$ to $n=1$ $\times 10^{18} \mathrm{~cm}^{-3}, \bar{\tau}_{L+}$ increases, while $\bar{\tau}_{L-}$ rapidly decreases. This is in fact appealing in serving for our purpose because, as mentioned previously, $\bar{\tau}_{L+}$ for the carrier-relevant $L+$ mode could be directly matched to the carrier Hall mobility. ${ }^{15}$ As $n$ approaches $n_{0}$, it is true that the $L+$ mode lives longer and longer. However, it is important to note that near $n \approx n_{0}$, the $L+$ mode will not be carrier relevant any more, but in fact just a half-half mixture of phonon and plasmon. In the case, $\bar{\tau}_{L_{+}}$could not carry the direct information of the carrier mobility. Therefore, practically one needs to be in the high density region away from $n_{0}$.

In Fig. 3, from the full many-body time-dependent calculation accounting for the mode coupling $g$, we displayed

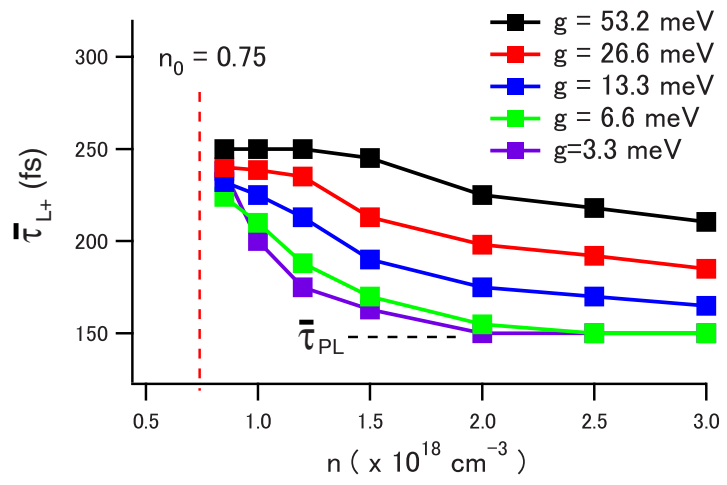

FIG. 3. (Color online) Relaxation time of $L+$ mode $\bar{\tau}_{L+}$ with respect to values of the mode coupling $g$ and the carrier density $n$. the behaviors of $\bar{\tau}_{L+}$ with respect to $n$ and $g$. As $g$ increases, $\bar{\tau}_{L+}$ generally increases. Most interestingly, in addition, there could be found a qualitative change in an increasing behavior of $\bar{\tau}_{L+}$. When $g$ is small (see $g=3.3 \mathrm{meV}$ ), $\bar{\tau}_{L+}$ is found to increase monotonically as $n$ approaches $n_{0}$, as pointed out by Kuznetsov and Stanton ${ }^{25}$ in terms of their classical treatment of lattice and electric field oscillation. On the other hand, when $g$ is large (see $g=13.3 \mathrm{meV}$ ), $\bar{\tau}_{L+}$ rapidly increases as $n$ decreases and gets a bump leading to the curvature change in the region of rather a high density $\left(>1 \times 10^{18} \mathrm{~cm}^{-3}\right)$ before $n$ reaches $n_{0}$, or when $g$ is much larger (see $g=26.6$ or $53.2 \mathrm{meV}), \bar{\tau}_{L+}$ arrives at a broad maximum near $n \sim 1.5$ $\times 10^{18} \mathrm{~cm}^{-3}$. This characteristic behavior of $\bar{\tau}_{L+}$ under a large mode coupling (for cases of $g=13.3,26.6$, and 53.2 $\mathrm{meV}$ ) is interesting and promising in view of the carrier mobility control because it proves possible to do over the wide value range of the carrier mobility in the high density region. Here we remember that $g$ can be controlled by $n_{\text {chem }}$ and $n$ $\left(=n_{\text {chem }}+n_{\text {opt }}\right)$ be controlled by $n_{\text {opt }}$, that is, both $g$ and $n$ are externally controllable.

We now search for the condition realizing a bump (of convex curvature) or maximum of $\bar{\tau}_{L+}$ before $n$ reaches $n_{0}$. Within the second order perturbation theory of the mode coupling $g, \gamma_{L+}$ and $\gamma_{L_{-}}$(their inverses give $\bar{\tau}_{L+}$ and $\bar{\tau}_{L_{-}}$, respectively) can be obtained in the limit of $\gamma_{\mathrm{PL}} \gg \gamma_{\mathrm{LO}}$,

$$
\begin{aligned}
& \gamma_{L+}=\left[1-\frac{g^{2}}{\left(\omega_{\mathrm{PL}}-\omega_{\mathrm{LO}}\right)^{2}+\gamma_{\mathrm{PL}}^{2}}\right] \gamma_{\mathrm{PL}}, \\
& \gamma_{L-}=\frac{g^{2}}{\left(\omega_{\mathrm{PL}}-\omega_{\mathrm{LO}}\right)^{2}+\gamma_{\mathrm{PL}}^{2}} \gamma_{\mathrm{PL}} .
\end{aligned}
$$

$\gamma_{L+}$ of Eq. (5) is of great interest. It immediately lets us know that there could be two qualitatively different behaviors of $\gamma_{L+}$ depending on the values of $g$. First, if $g<\gamma_{\mathrm{PL}}, \gamma_{L+}$ undergoes a monotonic increase as $n$ approaches $n_{0}$ like a case of $g=3.3 \mathrm{meV}$ in Fig. 3, which is consistent with Kuznetsov and Stanton. ${ }^{25}$ Let us denote $\gamma_{\mathrm{PL}}=4.4 \mathrm{meV}$ (i.e., $1 / \gamma_{\mathrm{PL}}$ 

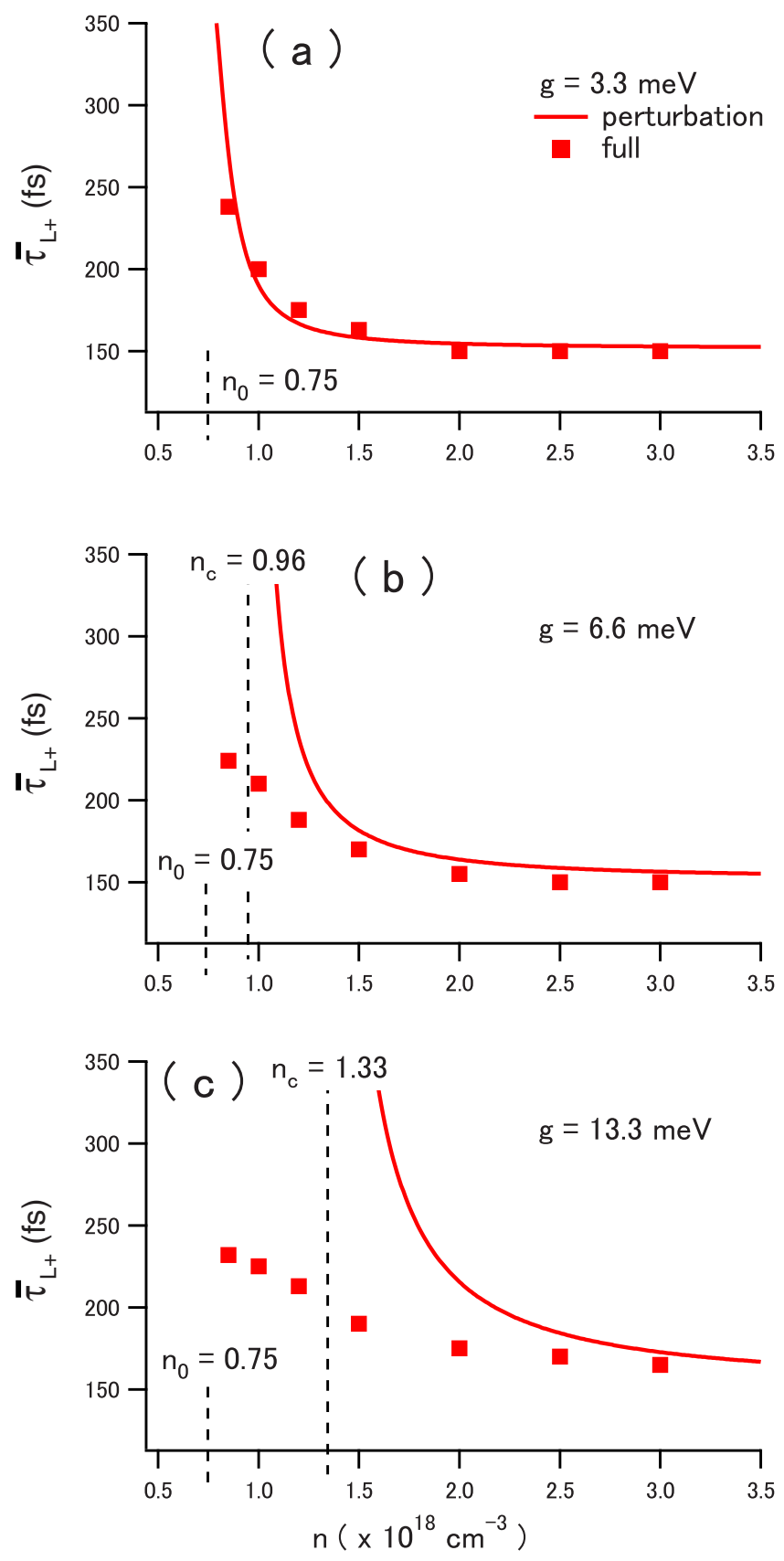

FIG. 4. (Color online) Comparison of the full many-body time-dependent calculation of $\bar{\tau}_{L+}$ with its second order perturbation results. (a) $g<\gamma_{\mathrm{PL}}$ and [(b) and (c)] $g>\gamma_{\mathrm{PL}}$.

$=150 \mathrm{fs}$ ) in our parameter set. Second, if $g>\gamma_{\mathrm{PL}}, \gamma_{L_{+}}$meets a dramatic situation leading to $\gamma_{L+} \rightarrow 0$ (i.e., $\bar{\tau}_{L+} \rightarrow \infty$ : the infinite relaxation time of $L+$ mode) at a certain critical density $n_{c}$ satisfying $\left(\omega_{\mathrm{PL}}-\omega_{\mathrm{LO}}\right)^{2}+\gamma_{\mathrm{PL}}^{2}=g^{2}$ according to Eq. (5). $n_{c}$ is the critical density leading to $\bar{\tau}_{L+} \rightarrow \infty$ within the second order perturbation theory. Explicitly, $n_{c}$ will be given by $n_{c}$ $=\left(\varepsilon_{\infty} m^{*} / 4 \pi\right)\left[\omega_{\mathrm{LO}}+\sqrt{g^{2}-\gamma_{\mathrm{PL}}^{2}}\right]^{2}$. Of course, this dramatic divergence of $\bar{\tau}_{L+}$ does not occur in a real situation of Fig. 3 because Eq. (5) is just from the second order perturbation theory which would not be so reliable as $n$ is near $n_{c}$. In Fig. 4 , we provide the comparison between the full many-body time-dependent calculation of $\bar{\tau}_{L+}$ and its second order perturbation results for two distinguishable cases of $g<\gamma_{\mathrm{PL}}$ and $g>\gamma_{\mathrm{PL}}$. Figure 4(a) with $g<\gamma_{\mathrm{PL}}$ shows that the full calcula-
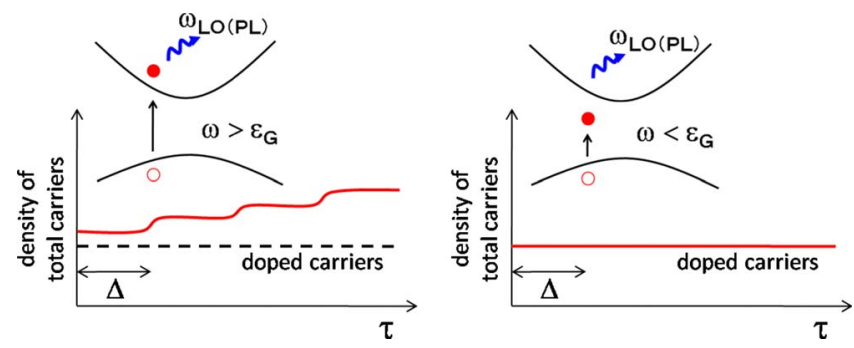

FIG. 5. (Color online) Excitation of real (left) and virtual (right) electronhole pair continuum. In contrast to the above-band-gap excitation case, the below-band-gap excitation case $\left(\omega<\epsilon_{G}\right)$ does not accumulate carriers (i.e., no photoexcited carrier). The figure is from Ref. 17.

tion result agrees well with the perturbation one. On the other hand, Figs. 4(b) and 4(c) with $g>\gamma_{\mathrm{PL}}$ show that the full calculation result deviates from the perturbation one, especially near $n \approx n_{c}$. Instead, it is found that the singular divergence of the perturbation result weakens and emerges as an appearance of a bump near $n \approx n_{c}$. Therefore, although the condition of $g>\gamma_{\mathrm{PL}}$ with the critical density $n_{c}$ cannot lead to a real singular divergence of $\bar{\tau}_{L+} \rightarrow \infty$, such conditions can be an important landmark for the actual ultrafast control of the carrier mobility through $\bar{\tau}_{L+}$. In conclusion, when $g$ $>\gamma_{\mathrm{pL}}$, we find that $\bar{\tau}_{L+}$ gets a bump or broad maximum reflecting the weak singular behavior of $\bar{\tau}_{L+}$ in the high density region characterized by $n \sim n_{c}$. If one realizes such a situation by finely balancing two kinds of carrier densities, i.e., $n_{\text {chem }}$ and $n_{\text {opt }}$, one could enhance the carrier mobility by a few tens of percent as shown in Fig. 3.

\section{B. Second option: Optimization of optical pumping pulse}

In this option, ${ }^{17}$ we require a series of optical pumping pulses prepared in a special way. It should be prepared to have a form of the pulse train with an interval between pulses $\Delta . \bar{\Theta}(\tau)$ in Eq. (1) should then correspond to $\bar{\Theta}(\tau)$ $=\sum_{n=0}^{n=N-1}[\Theta(\tau+n \Delta)-\Theta(\tau+n \Delta-\sigma)]$ for a pulse train with $N$ pulses. We define $\delta$ ( $\equiv \epsilon_{G}-\omega ; \epsilon_{G}$ : energy gap) as the detuning of the laser with respect to the electron-hole continuum, so that $\delta>0$ signifies the below-band-gap excitation. An application of the below-band-gap excitation pulses introduces the virtual electron-hole continuum in a semiconductor, which excites phonons and plasmons. It is a pure quantum mechanical feature, which is sharply in contrast with the classical description. ${ }^{25}$ The manipulation of coherent phonons and plasmons using the virtual electron-hole pair avoids the dephasing processes. First, it does not accumulate the photoexcited (or optically doped) carriers in a conduction band, as shown in Fig. 5, i.e., $n=n_{\text {chem. }}$ Otherwise, there would be significant damping of coherent phonon and plasmon due to scattering with optically generated electrons and holes. ${ }^{30}$ Second, no change in the carrier density suppresses the free-streaming current of the counterdirected carrier stream by succeeding pulses. ${ }^{31}$ Third, when a semiconductor is illuminated by a laser with an electric field $E_{\alpha}$, the field energy $\mathcal{E}$ stored in the semiconductor is $\mathcal{E}=$ $-\Sigma_{\alpha \beta} \chi_{\alpha \beta}(\omega) E_{\alpha} E_{\beta}$ with the optical response function $\chi_{\alpha \beta}(\omega)$. 

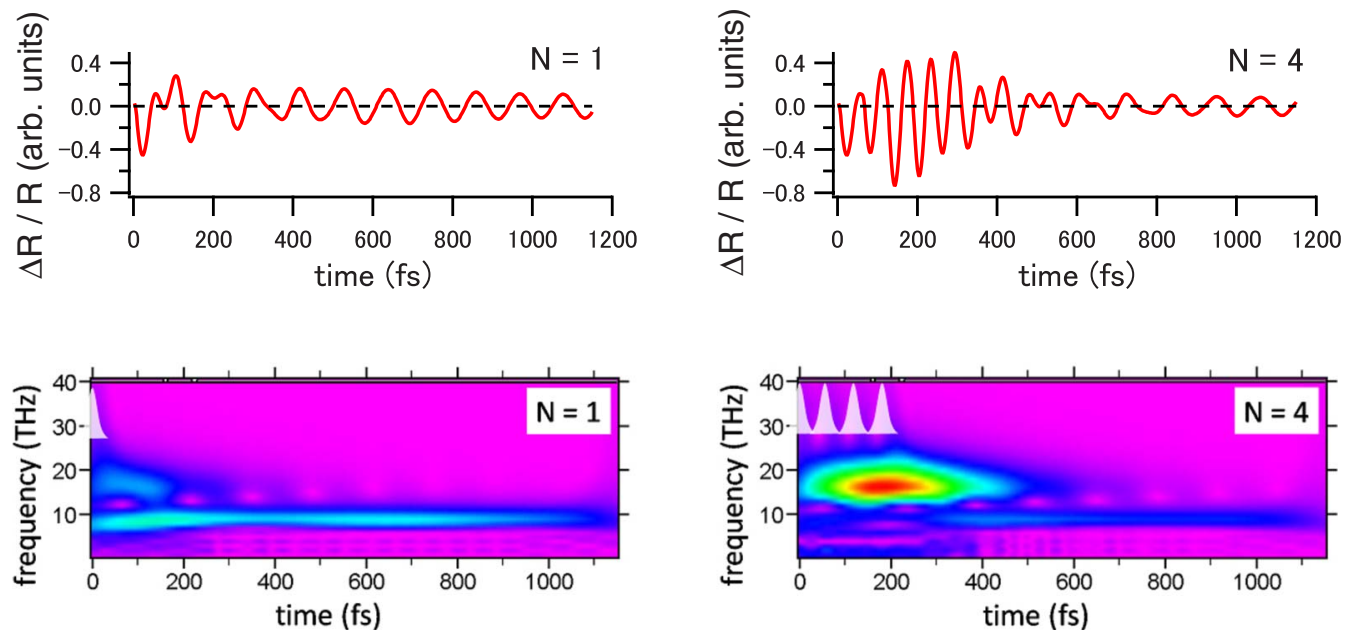

FIG. 6. (Color online) Upper panel: transient electro-optic responses $\Delta R / R\left[\propto P_{L}(\tau)+P_{E}(\tau)\right.$ with $\left.r_{L} / r_{E}=1\right]$ for the pulse train of $N=1$ and $N=4$. Lower panel: continuous wavelet transformation of $\Delta R / R$ in the frequency-time space. The density of doped carriers is $n=2\left(\times 10^{18} \mathrm{~cm}^{-3}\right)$ and the interval between pulses is $\Delta=2 \pi / \omega_{L+}$, i.e., $61 \mathrm{fs}$. The detuning $\delta=40 \mathrm{meV}$ is adopted. The applied pulse train is illustrated.

For $\omega<\epsilon_{G}$, the dissipative part $\operatorname{Im}\left[\chi_{\alpha \beta}(\omega)\right]$ would be zero, so that the spontaneous emission is suppressed. ${ }^{18}$

In the upper panel of Fig. 6, transient electro-optic responses $\Delta R / R$ are provided as $\Delta R / R \propto P_{L}(\tau)+P_{E}(\tau)$ by simply taking $r_{L} / r_{E}=1$. In the frequency-time space of the lower panel of Fig. 6, it is clear that the oscillations of $\Delta R / R$ are composed of two modes, i.e., $L+$ (higher frequency) and $L$ - (lower frequency) modes. To be most interesting, the pulse train with $N=4$ is found to appreciably sustain the coherent oscillation of $L+$ mode compared to a single pulse $(N=1)$. For a single pulse, there would be no qualitative difference between handlings with real and virtual excitations [compare with Fig. 1(c)].

It is our main interest to investigate how to control the coherent motion of $L+$ mode because it is directly connected to the carrier mobility of a semiconductor through $\mu$ $\approx e \bar{\tau}_{L+} / m^{*}{ }^{16}$ In Fig. 7 , adopting the $N=8$ pulse train, we displayed the coherent motion of both $L+$ and $L-$ modes as the carrier density $n$ changes from $1.5 \times 10^{18}$ to 3 $\times 10^{18} \mathrm{~cm}^{-3}$ for the pulse interval $\Delta=2 \pi / \omega_{L+}$. The frequency of $L+$ mode shifts up as $n$ increases, while the coherent motion of $L+$ mode survives longer as $n$ decreases just as found in Sec. II. In Fig. 8, the dynamics of LOPC modes is illustrated with changing the pulse interval $\Delta$ from 45 to 100 fs for a fixed carrier density $n=2 \times 10^{18} \mathrm{~cm}^{-3}$. In the figure, the well-defined coherent motion of $L+$ mode continues up to $\sim 700$ fs at $\Delta=60 \mathrm{fs}$, but the coherence of $L+$ mode rapidly disappears at the slightly different interval like $\Delta=50$ or 70 fs so that its coherent motion cannot continue that long. It is immediately noted that $\Delta=60 \mathrm{fs}$ is almost the oscillation period of $L+$ mode, $2 \pi / \omega_{L+}\left(=61\right.$ fs for $\left.n=2 \times 10^{18} \mathrm{~cm}^{-3}\right)$. This implies that in order to control the coherent motion of $L+$ mode, it is essential to synchronize the pulse interval $\Delta$ with the oscillation period of $L+$ mode. In Fig. 9(a), we provide the behavior of $\bar{\tau}_{L+}$ with respect to $\Delta$ for the $N=8$ pulse train. ${ }^{32}$ It is clear that $\Delta=2 \pi / \omega_{L+}$ makes possible the most efficient control of the coherent motion of $L+$ mode. Further, for an effective control in the case, $\Delta$ should be synchronized within $\sim \pm 5$ fs around $2 \pi / \omega_{L+}$. The longest coherence time of $\sim 700$ fs at $\Delta=60$ fs enables us to estimate the largest carrier mobility of $18377 \mathrm{~cm}^{2} \mathrm{~V} \mathrm{~s}$, which is almost twice of the maximum mobility obtained in $n$-GaAs at $300 \mathrm{~K}$ with doping density of $\sim 10^{15} \mathrm{~cm}^{-3}{ }^{33}$ Note that such large mobility can be obtained in $n$-GaAs only at low temperature, like $180 \mathrm{~K}$. This implies a possibility of laser cooling of semiconductors since the below-band-gap excitation can remove thermal energy. ${ }^{34}$

Finally, returning to the important premise of the present study, we note that it is highly nontrivial to guarantee the virtual electron-hole continuum using the below-band-gap excitation scheme. As a matter of fact, the positive detuning

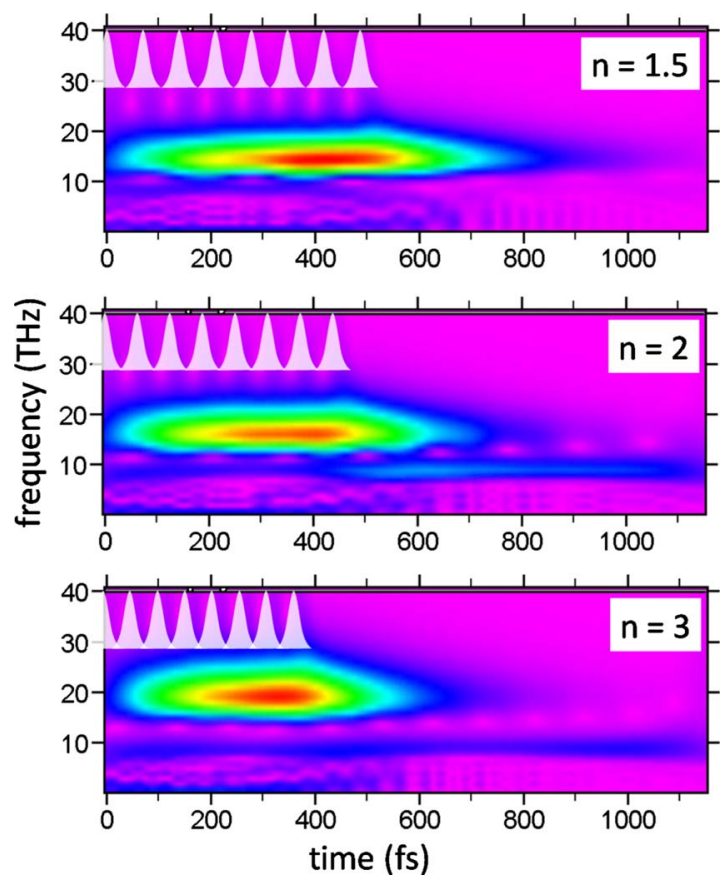

FIG. 7. (Color online) Continuous wavelet transformation of $\Delta R / R$ in the frequency-time space for the pulse train of $N=8 . \Delta R / R$ with respect to the densities of doped carrier, $n=1.5,2$, and $3\left(\times 10^{18} \mathrm{~cm}^{-3}\right)$ for a fixed pulse interval, $\Delta=2 \pi / \omega_{L+} . \delta=40 \mathrm{meV}$ is taken. The applied pulse train is illustrated in each panel. 

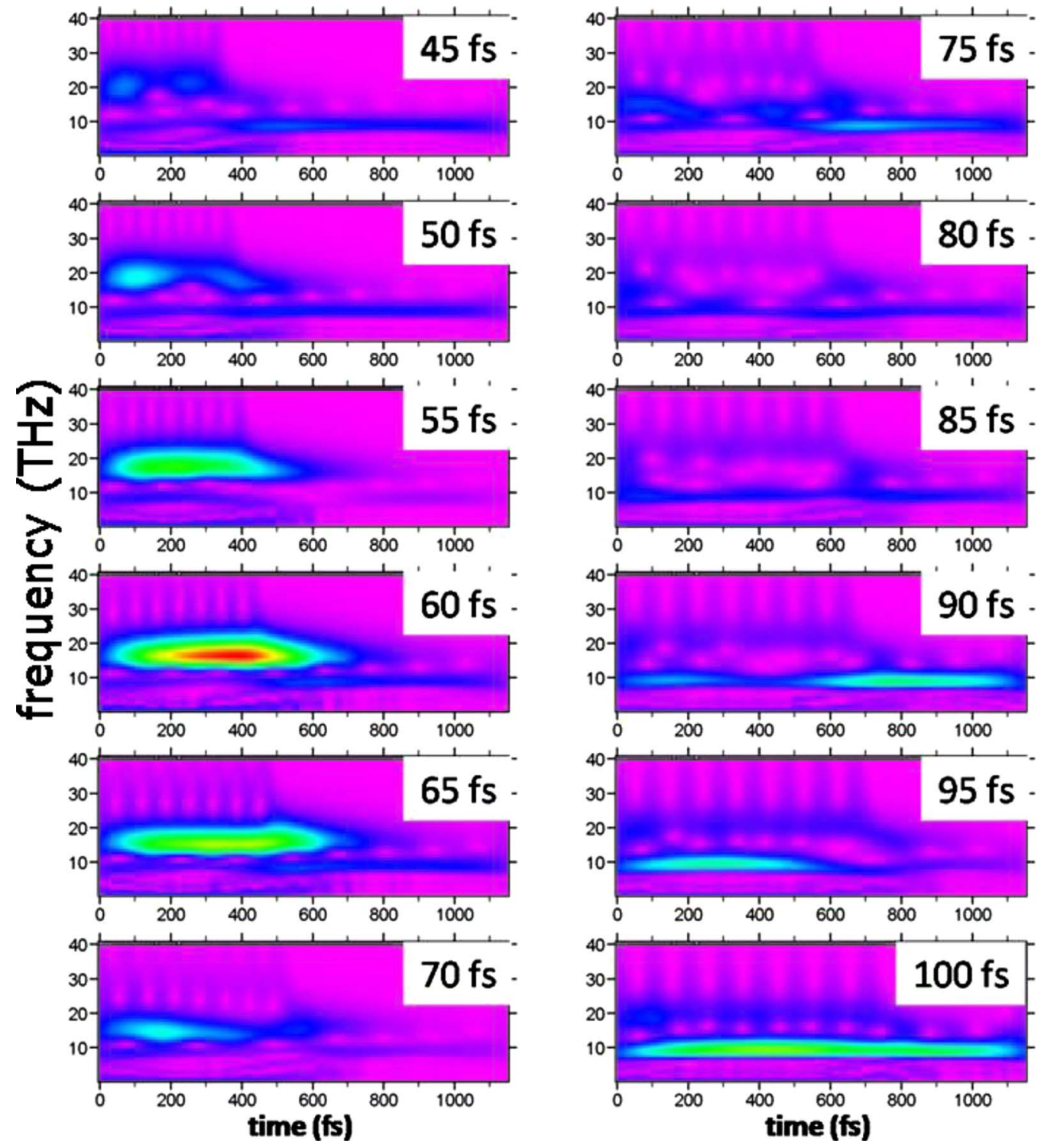

FIG. 8. (Color online) Continuous wavelet transformation of $\Delta R / R$ in the frequency-time space for the pulse train of $N=8$. $\Delta R / R$ with respect to the intervals between pulses, $\Delta=45-100$ fs for a fixed density of doped carrier, $n=2\left(\times 10^{18} \mathrm{~cm}^{-3}\right) . \delta=40 \mathrm{meV}$ is taken.

( $\delta>0$, i.e., $\left.\omega<\epsilon_{G}\right)$ is not sufficient to guarantee the virtual excitation because the pulse in principle includes all the frequency components within the broad pulse spectra, and there are band tail and excitonic absorption just below the gap. ${ }^{35}$
One needs an additional condition regulating the pulse shape or strength. The consideration can be reduced to the dynamics between two levels of the real excited state $|r . e x\rangle$ and the virtual excited state $|v . e x\rangle$, that is, $i(\partial / \partial \tau) C_{v . e x}(\tau)$
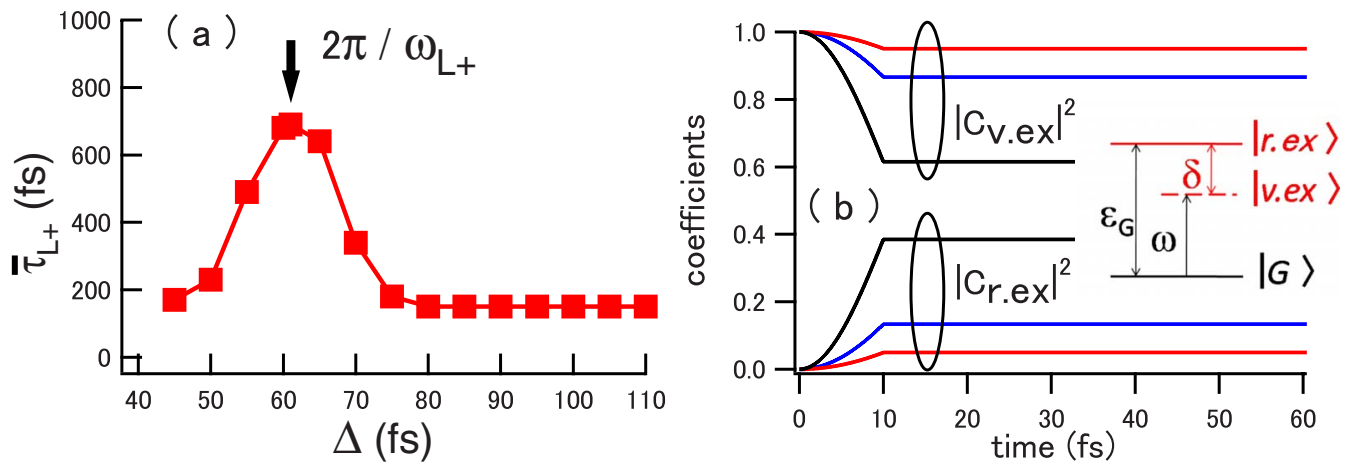

FIG. 9. (Color online) (a) Behaviors of $\bar{\tau}_{L+}$ with respect to $\Delta$ for the pulse train of $N=8 \cdot n=2\left(\times 10^{18} \mathrm{~cm}^{-3}\right)$ and $\delta=40$ meV are taken. (b) Behaviors of $\left|C_{\text {v.ex }}(\tau)\right|^{2}$ and $\left|C_{\text {r.ex }}(\tau)\right|^{2}$ in the simplified two-level problem for $\Omega_{0}=15 \mathrm{meV}$ (red), $25 \mathrm{meV}$ (blue), and $45 \mathrm{meV}$ (black). $\sigma=10 \mathrm{fs}$ and $\delta=40 \mathrm{meV}$ are adopted. The inset is the level schematic. $|G\rangle$ is the ground state. The figure is from Ref. 17. 
$=\Omega(\tau) C_{\text {r.ex }}(\tau)$ and $i(\partial / \partial \tau) C_{\text {r.ex }}(\tau)=\delta C_{\text {r.ex }}(\tau)+\Omega(\tau) C_{\text {v.ex }}(\tau)$, where $\Omega(\tau)$ depicts the pulse shape, $\Omega(\tau)=\Omega_{0}[\Theta(\tau)-\Theta(\tau$ $-\sigma)$ ], and we have $C_{v . e x}(0)=1$ and $C_{\text {r.ex }}(0)=0$. From Fig. 9 (b), it is found that the laser light with the weak field amplitude (say $\ll 15 \mathrm{meV}$ ) could be applicable for our purpose. This situation can be also described by the adiabatic Hamiltonian

$$
\mathcal{H}_{0}(\tau)=\left(\begin{array}{cc}
0 & \Omega(\tau) \\
\Omega(\tau) & \delta
\end{array}\right) .
$$

Here we require the condition that the time evolution of $|v . e x\rangle$ should induce the mixing with $|r . e x\rangle$ adiabatically, that is, $|\langle r . e x ; \tau|(\partial / \partial \tau|v . e x ; \tau\rangle)| \ll \delta .|v . e x ; \tau\rangle$ and $|r . e x ; \tau\rangle$ adiabatically evolve starting from $|v . e x\rangle$ and $|r . e x\rangle$, respectively. Keeping only the dominant term, we have $|\dot{\Omega}(\tau) \delta|$ $\ll \delta\left[\delta^{2}+\Omega(\tau)^{2}\right]$. From $\dot{\Omega}(\tau) \approx 2 \Omega_{0} / \sigma$, we finally have $\Omega_{0}$ $\ll \sigma \delta^{2} / 2$. This is in fact same as the adiabaticity condition in the optical control of spin quantum dots. ${ }^{18}$ Adopting $\sigma$ $=10 \mathrm{fs}$ and $\delta=40 \mathrm{meV}$, the pulse strength should satisfy $\Omega_{0} \ll 10 \mathrm{meV}$ according to the condition. $\Omega_{0} \rightarrow 0$ in our present study satisfies the required condition well.

\section{SUMMARY}

A control of the carrier mobility in a polar semiconductor is suggested based on the ultrafast dephasing of the coherent carrier-relevant LOPC mode ( $L+$ mode). Incorporating the idea, we have proposed two possible theoretical options to achieve the nontrivial control of the semiconductor mobility over its wide range of values. One option is to optimize the semiconductor. One may optimally fix the strength of phonon-plasmon coupling $g$ by adjusting the chemical doping density $n_{\text {chem }}$ and drive the (weak) singular dynamics of $L+$ mode by controlling the total carrier density $n$ ( $=n_{\text {chem }}+n_{\text {opt }}$, i.e., through optical doping) for a fixed $g$. When $g>\gamma_{\mathrm{PL}}$, in particular, we find that the relaxation time of $L+$ mode $\bar{\tau}_{L+}$ can be weakly singular and thus get a bump or a broad maximum in the high density region $n \sim n_{c}$ (away from $n_{0}$ ). This scheme could enhance the mobility by a few tens of percent. The other option is to optimize the optical pumping laser. In this option, the dynamics of coherent modes is driven by the virtual electron-hole continuum using the pumping pulse train of below-band-gap excitation (i.e., $\left.n=n_{\text {chem }}\right)$, which avoids the dephasing processes and then makes the coherent motion of the carrier-relevant $L+$ mode survive drastically long under the synchronization of the pulse train with its coherent oscillation. This implies that the carrier mobility $\mu \approx e \bar{\tau}_{L+} / m^{*}$ can be efficiently controlled and dramatically increased, which can be applied also to wide-gap semiconductors such as $\mathrm{GaN}, \mathrm{SiC}, \mathrm{InN}$, and their nanostructures.

\section{ACKNOWLEDGMENTS}

This work was supported by the Special Coordination Funds for Promoting Science and Technology from MEXT, Japan.

${ }^{1}$ A. Mooradian and G. B. Wright, Phys. Rev. Lett. 16, 999 (1966).

${ }^{2}$ G. D. Mahan, Many-Particle Physics (Plenum, New York, 1986).

${ }^{3}$ G. C. Cho, T. Dekorsy, H. J. Bakker, R. Hövel, and H. Kurz, Phys. Rev. Lett. 77, 4062 (1996).

${ }^{4}$ J. Shah, Ultrafast Spectroscopy of Semiconductors and Semiconductor Nanostructures (Springer-Verlag, Berlin, 1999).

${ }^{5}$ M. Hase, S. Nakashima, K. Mizoguchi, H. Harima, and K. Sakai, Phys. Rev. B 60, 16526 (1999).

${ }^{6}$ M. Hase, K. Ishioka, M. Kitajima, and K. Ushida, Appl. Phys. Lett. 82, 3668 (2003).

${ }^{7}$ H. Altan, X. Xin, D. Matten, and R. R. Alfano, Appl. Phys. Lett. 89, 052110 (2006).

${ }^{8}$ R. Huber, C. Kübler, S. Tübel, A. Leitenstorfer, Q. T. Vu, H. Haug, F. Köhler, and M. C. Amann, Phys. Rev. Lett. 94, 027401 (2005).

${ }^{9}$ G. O. Smith, T. Juhasz, W. E. Bron, and Y. B. Levinson, Phys. Rev. Lett. 68, 2366 (1992).

${ }^{10}$ Y. M. Chang, C. T. Chuang, C. T. Chia, K. T. Tsen, H. Lu, and W. J. Schaff, Appl. Phys. Lett. 85, 5224 (2004).

${ }^{11}$ Y. M. Chang, H. W. Chu, C. H. Shen, and S. Gwo, Appl. Phys. Lett. 90, 072111 (2007).

${ }^{12}$ T. Dekorsy, H. Auer, H. J. Bakker, H. G. Roskos, and H. Kurz, Phys. Rev. B 53, 4005 (1996).

${ }^{13}$ K. Liu, J. Xu, T. Yuan, and X. C. Zhang, Phys. Rev. B 73, 155330 (2006).

${ }^{14}$ M. C. Beard, G. M. Turner, and C. A. Schmuttenmaer, Phys. Rev. B 62, 15764 (2000)

${ }^{15}$ S. Nakashima and H. Harima, Phys. Status Solidi A 162, 39 (1997).

${ }^{16}$ M. Hase, Phys. Status Solidi C 5, 364 (2008); M. Hase, Appl. Phys. Lett. 94, 112111 (2009).

${ }^{17}$ J. D. Lee and M. Hase, Phys. Rev. Lett. 101, 235501 (2008).

${ }^{18}$ C. Piermarocchi, P. Chen, L. J. Sham, and D. G. Steel, Phys. Rev. Lett. 89, 167402 (2002)

${ }^{19}$ I. V. Mel'nikov, D. Mihalache, F. Moldoveanu, and N. C. Panoiu, Phys. Rev. A 56, 1569 (1997).

${ }^{20}$ A. Mysyrowicz, D. Hulin, A. Antonetti, A. Migus, W. T. Masselink, and H. Morkoc, Phys. Rev. Lett. 56, 2748 (1986).

${ }^{21}$ S. Schmitt-Rink and D. S. Chemla, Phys. Rev. Lett. 57, 2752 (1986).

${ }^{22}$ T. Unold, K. Mueller, C. Lienau, T. Elsaesser, and A. D. Wieck, Phys. Rev. Lett. 92, 157401 (2004).

${ }^{23}$ B. I. Lundqvist, Phys. Kondens. Mater. 6, 193 (1967).

${ }^{24}$ J. D. Lee, J. Inoue, and M. Hase, Phys. Rev. Lett. 97, 157405 (2006).

${ }^{25}$ A. V. Kuznetsov and C. J. Stanton, Phys. Rev. B 51, 7555 (1995).

${ }^{26}$ Phonons and plasmons would relax mainly due, respectively, to anharmonic and carrier-carrier scattering.

${ }^{27}$ In terms of $\mu=e \bar{\tau} / m^{*}, \bar{\tau}=150$ fs corresponds to the carrier mobility of $\sim 8000 \mathrm{~cm}^{2} \mathrm{~V} \mathrm{~s}$ in $n$-GaAs with the doping density of $\sim 1 \times 10^{15} \mathrm{~cm}^{-3}$ at $300 \mathrm{~K}$.

${ }^{28}$ B. A. Sanborn, Phys. Rev. B 51, 14256 (1995).

${ }^{29}$ R. Fukasawa and S. Perkowitz, Phys. Rev. B 50, 14119 (1994).

${ }^{30}$ M. P. Hasselbeck, D. Stalnaker, L. A. Schlie, T. J. Rotter, A. Stintz, and M. Sheik-Bahae, Phys. Rev. B 65, 233203 (2002).

${ }^{31}$ B. I. Bakunov, A. V. Maslov, and S. N. Zhukov, Phys. Rev. B 67, 153201 (2003).

${ }^{32}$ Because the temporal profile of $L+$ mode is not relaxational, the definition of $\bar{\tau}_{L+}$ is rather arbitrary. For Fig. 8(a), $\bar{\tau}_{L+}$ is defined as the time that the intensity gets less than about $40 \%$ of the maximum intensity at $\Delta$ $=2 \pi / \omega_{L+}$. If the coherent motion of $L+$ mode is suppressed enough, $\bar{\tau}_{\mathrm{PL}}$ $(=150 \mathrm{fs})$ is simply assigned to $\bar{\tau}_{L+}$.

${ }^{33}$ G. E. Stillman, C. M. Wolfe, and J. O. Dimmock, J. Phys. Chem. Solids 31, 1199 (1970)

${ }^{34}$ M. Sheik-Bahae and R. I. Epstein, Phys. Rev. Lett. 92, 247403 (2004).

${ }^{35}$ A. Leitenstorfer, A. Lohner, T. Elsaesser, S. Haas, F. Rossi, T. Kuhn, W. Klein, G. Boehm, G. Traenkle, and G. Weiman, Phys. Rev. Lett. 73, 1687 (1994). 